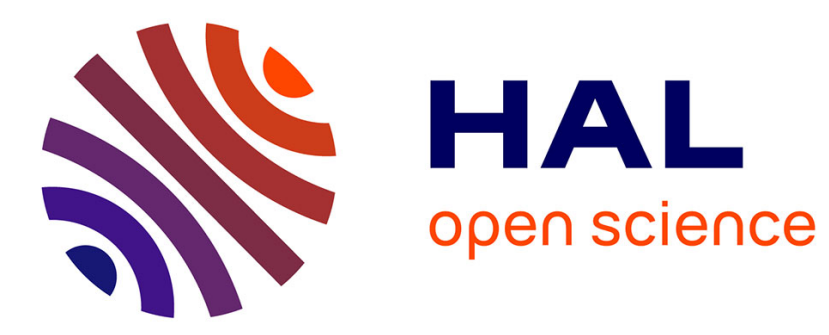

\title{
A Simple Integral Formulation for the Modeling of Thin Conductive Shells
}

\author{
Tung Le Duc, Gérard Meunier, Olivier Chadebec, Jean-Michel Guichon, \\ Laurent Krähenbühl
}

\section{> To cite this version:}

Tung Le Duc, Gérard Meunier, Olivier Chadebec, Jean-Michel Guichon, Laurent Krähenbühl. A Simple Integral Formulation for the Modeling of Thin Conductive Shells. European Physical Journal: Applied Physics, 2013, 64 (02), 24513 (5 p.). 10.1051/epjap/2013120413 . hal-00819093

\section{HAL Id: hal-00819093 https://hal.science/hal-00819093}

Submitted on 11 Dec 2020

HAL is a multi-disciplinary open access archive for the deposit and dissemination of scientific research documents, whether they are published or not. The documents may come from teaching and research institutions in France or abroad, or from public or private research centers.
L'archive ouverte pluridisciplinaire HAL, est destinée au dépôt et à la diffusion de documents scientifiques de niveau recherche, publiés ou non, émanant des établissements d'enseignement et de recherche français ou étrangers, des laboratoires publics ou privés. 


\title{
A simple integral formulation for the modeling of thin conductive shells ${ }^{\star}$
}

Tung Le-Duc ${ }^{1, \text { a }}$, Gérard Meunier ${ }^{2}$, Olivier Chadebec ${ }^{2}$, Jean-Michel Guichon² ${ }^{2}$, and Laurent Krähenbühl ${ }^{3}$

${ }^{1}$ Department of Electric Power Systems, School of Electrical Engineering, Hanoi University of Science and Technology, 1 Dai Co Viet Road, 1000 Hanoi, Vietnam

${ }^{2}$ Grenoble Electrical Engineering Laboratory, University of Grenoble Grenoble-INP/Université Joseph Fourier/CNRS UMR 5269, 11 rue des Mathématiques, BP 46, 38402 Saint-Martin-d'Héres Cedex, France

${ }^{3}$ Université de Lyon, AMPERE, CNRS UMR 5005, École Centrale de Lyon, 38 av. Guy de Collongue, 69134 Ecully Cedex, France

\begin{abstract}
In order to model thin conductive non-magnetic shells, an original surface integral formulation is proposed. The method is based on a surface impedance condition which takes into account the field variation through depth due to skin effect. It is general and enables the modeling of various problems whatever their skin depth and avoiding the meshing of the air region. The formulation is compared with another integral formulation recently proposed by authors and is validated thanks to an axisymmetric finite-element method (FEM). Advantages and drawbacks of this new formulation are discussed.
\end{abstract}

\section{Introduction}

Thin conductive non-magnetic shells are frequently encountered in electromagnetic devices (such as planar conductors and conductive shieldings) and the analysis of their eddy-current distributions is an important problem in engineering. The geometric specificity of a thin region is characterized by a high ratio between the length and the thickness, so using a volume mesh leads to a large number of elements. Furthermore, when frequency is high, skin depth can become much thinner than thickness $e$, leading to an increase of the size of the mesh if this effect has to be properly modeled. Shell element formulations have been developed to overcome such difficulties, e.g., with the boundary element method (BEM) [1], with the finite-element method (2D formulation [2] and $3 \mathrm{D}$ formulation [3-8]), and with an integral method recently, proposed by the authors of this paper [9].

In [9], a general shell element formulation for modeling thin conductive regions has been proposed $(\delta \gg e$ or $\delta \approx e$ or $\delta \ll e)$. Like in [1-8], this element takes into account the field variation through depth due to skin effect. The shell is modeled by an integral formulation avoiding the mesh of air region. Based on a simple surface discretization of the shell, the number of unknowns considerably reduces, the depth of the shell being not meshed.

The present method is a natural reduction of the method proposed in [9]. However, we will see that the formulation proposed in this paper is less consuming in terms of degrees of freedom than in [9] and the complex computation of volume singular integrals is avoided. On the other hand, the computation of eddy-current losses is not possible so this new formulation is reliable for applications where only magnetic field computation in the air region is focused.

In Section 2, the integral equation governing the thin shell regions will be presented. In Section 3, this equation will be coupled with a surface integral formulation. Finally, two numerical examples will be proposed in the last part. Results obtained with our formulation will be compared with those given in [9] and the finite-element method (FEM).

\section{Formulation}

\subsection{An equation for the thin region}

We consider that a small skin depth $\delta$ is associated to the non-magnetic shell with a thickness e (Fig. 1). The field

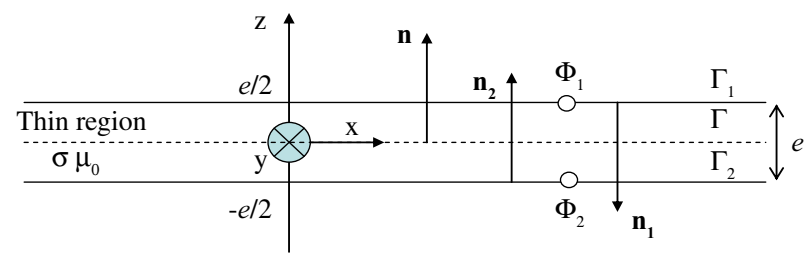

Fig. 1. Thin conductive region. 
variation of the longitudinal component across thickness of shell can be approximated by the analytical solution of the problem for an infinite plane $[1,3]$ :

$\mathbf{H}_{s}(z)=\frac{1}{\operatorname{sh}(a e)}\left[\mathbf{H}_{1 s} \operatorname{sh}\left(\frac{a e}{2}+a z\right)+\mathbf{H}_{2 s} \operatorname{sh}\left(\frac{a e}{2}-a z\right)\right]$,

where $a=(1+j) / \delta, \mathbf{H}_{1 s}$ and $\mathbf{H}_{2 s}$ are the field tangential values on both sides of the shell. Applying Galerkin method on the Maxwell-Faraday equations for side "1" of the shell on a real $3 \mathrm{D}$ surface $\Gamma_{1}$ of the shell, we get [3]:

$$
\int_{\Gamma_{1}} \operatorname{grad}_{s} w \cdot\left(\alpha \mathbf{H}_{1 s}-\beta \mathbf{H}_{2 s}\right) \mathrm{d} \Gamma+j \omega \int_{\Gamma_{1}} w \mathbf{B}_{1} \cdot \mathbf{n}_{1} \mathrm{~d} \Gamma=0,
$$

where $\alpha=\frac{a}{\sigma t h(a e)}, \beta=\frac{a}{\sigma s h(a e)} ; w$ is a set of nodal surface weighting function; $\mathbf{n}_{1}$ is the normal vector corresponding to the side "1" of the shell.

The other equation corresponding to the other side of the shell is obtained when indices " 1 " and "2" are exchanged:

$$
\int_{\Gamma_{2}} \operatorname{grad}_{s} w \cdot\left(\alpha \mathbf{H}_{2 s}-\beta \mathbf{H}_{1 s}\right) \mathrm{d} \Gamma+j \omega \int_{\Gamma_{2}} w \mathbf{B}_{2} \cdot \mathbf{n}_{2} \mathrm{~d} \Gamma=0,
$$

where $\mathbf{n}_{2}$ is the normal vector corresponding to the side "2" of the shell.

Equations (2) and (3) represent the electromagnetic behavior of the conductive shell itself. In order to take into account the surrounding air region, it remains to couple both equations. In [2-8], the authors choose to use the finite-element method (FEM), thus they need to mesh the air region. In [1], a boundary element method (BEM) is preferred but the authors have to manage the coupling on both sides of the shell and have to determine if both sides of the shell are interfaced with a single region or with two different ones. This leads to the need of quite sophisticated geometrical analysis tools if the method has to be applied to general geometries. Moreover, four unknowns per node are needed. In [9], we have developed an integral approach based on the coupling of (2) and (3) with a volume integral equation. The obtained formulation presents an interesting compactness treating different air volumes as the single physical region. With this method two unknowns per node are needed but the computation of volume integrals can become very complicated to accurately integrate the exponential decreasing of the current across the thickness especially when the contribution of an element on itself is considered. This integration is quite hard to achieve and is certainly the weak point of the method.

In this work, a new approach is proposed, based on the coupling of (2) and (3) with a simpler surface integral equation. This coupling only leads to one unknown per node and is presented in the next section.

\subsection{Surface integral formulation}

Equations (2) and (3) are written on the averaged surface $\Gamma$ of the thin region. Subtracting equations (3) and (2) leads to:

$$
\begin{gathered}
(\alpha+\beta) \int_{\Gamma} \operatorname{grad}_{s} w \cdot\left(\mathbf{H}_{2 s}-\mathbf{H}_{1 s}\right) \mathrm{d} \Gamma \\
+j \omega \int_{\Gamma} w(2 \cdot \mathbf{B} \cdot \mathbf{n}) \mathrm{d} \Gamma=0,
\end{gathered}
$$

where $\mathbf{B} \cdot \mathbf{n}=\frac{1}{2}\left(\mathbf{B}_{2} \cdot \mathbf{n}_{2}-\mathbf{B}_{1} \cdot \mathbf{n}_{1}\right)$ is the averaged normal induction on the surfaces $\Gamma_{1}$ and $\Gamma_{2}$.

Let us now assume that the shell is placed in an inductor field $\mathbf{H}_{\mathrm{o}}$. The total magnetic field $\mathbf{H}$ is the sum of $\mathbf{H}_{\mathrm{o}}$ and $\mathbf{H}_{\mathrm{r}}$, the reaction field of the eddy current in thin shell:

$$
\mathbf{H}=\mathbf{H}_{\mathrm{O}}+\mathbf{H}_{\mathrm{r}} \text {. }
$$

A reduced magnetic scalar potential is introduced for each side of the shell. Since there is a jump of the tangential component of the magnetic field through the element, a double layer node surface element is needed [9]. Expressions of tangential magnetic fields on both sides and outside the shell are:

$$
\mathbf{H}_{1 s}=\mathbf{H}_{\mathrm{os}}-\operatorname{grad}_{s} \phi_{1} \quad \mathbf{H}_{2 s}=\mathbf{H}_{\mathrm{os}}-\operatorname{grad}_{s} \phi_{2},
$$

where $\mathbf{H}_{\mathrm{os}}$ is the tangential source field and $\phi_{1}$ and $\phi_{2}$ represent the magnetic reduced scalar potential on each side of the shell.

Using (4) and (6), we get:

$(\alpha+\beta) \int_{\Gamma} \operatorname{grad}_{s} w \cdot \operatorname{grad}_{s} \delta \phi \mathrm{d} \Gamma+2 j \omega \int_{\Gamma} w \cdot \mathbf{B} \cdot \mathbf{n} \mathrm{d} \Gamma=0$,

where $\delta \phi=\phi_{1}-\phi_{2}$ is the discontinuity of scalar magnetic potential.

The equivalent surface shell current $\mathbf{K}$ can be expressed as in [1]:

$$
\mathbf{K}=\int_{e} \mathbf{J}(z) d z=\mathbf{n} \times \operatorname{grad}_{s} \delta \phi .
$$

Thanks to Biot and Savart law, we can write:

$$
\mathbf{H}_{r}=\frac{1}{4 \pi} \int_{\Gamma} \int_{e} \frac{\mathbf{J} \times \mathbf{r}}{r^{3}} \mathrm{~d} z \mathrm{~d} \Gamma \approx \frac{1}{4 \pi} \int_{\Gamma} \frac{\mathbf{K} \times \mathbf{r}}{r^{3}} \mathrm{~d} \Gamma .
$$

Using (6), (8) and (9), equation (7) becomes:

$$
\begin{aligned}
& (\alpha+\beta) \int_{\Gamma} \operatorname{grad}_{s} w \cdot \operatorname{grad} \delta \phi \mathrm{d} \Gamma+ \\
& \frac{2 j \omega \mu_{o}}{4 \pi} \int_{\Gamma} w \cdot \int_{\Gamma} \frac{\mathbf{n} \times \operatorname{grad} \delta \phi \times \mathbf{r}}{r^{3}} \mathrm{~d} \Gamma \cdot \mathbf{n} \mathrm{d} \Gamma \\
& =-2 j \omega \mu_{0} \int_{\Gamma} w \cdot \mathbf{H}_{o} \cdot \mathbf{n} \mathrm{d} \Gamma .
\end{aligned}
$$

This equation has now to be discretized. The easiest way is to mesh the average surface $\Gamma$, situated halfway between boundaries of the shells (Fig. 1), into $n$ triangular 
elements associated to a uniform tangential component of the eddy current (meaning first-order shape functions for the potential). The algebraic linear system obtained has $2 p$ unknowns (one complex magnetic scalar potential per node; the mesh being composed of $p$ nodes). Let us notice that shape functions with higher orders can be used.

The discontinuity of scalar magnetic potential is expressed as follows:

$$
\delta \phi=\sum_{k=1}^{p} w_{k}(x, y, z) \delta \phi_{k} .
$$

The obtained matrix system is the following one:

$$
[\mathbf{A}] \cdot[\delta \Phi]-[\mathbf{B} s] \cdot[\delta \Phi]=\left[\mathbf{h}_{0 n}\right],
$$

where

$$
\begin{gathered}
\mathbf{A}(i, k)=(\alpha+\beta) \int_{\Gamma} \operatorname{grad} w_{i} \cdot \operatorname{grad} w_{k} \mathrm{~d} \Gamma \\
\mathbf{B}_{s}(i, k)=\frac{2 j \omega \mu_{o}}{4 \pi} \int_{\Gamma} w_{i}\left(\int_{\Gamma_{k}} \frac{\mathbf{n} \times \operatorname{grad} w_{k} \times \mathbf{r}}{r^{3}} \mathrm{~d} \Gamma\right) \cdot \mathbf{n}_{i} \mathrm{~d} \Gamma
\end{gathered}
$$

$$
\mathbf{h}_{0 n}(i)=-2 j \omega \mu_{0} \int_{\Gamma} w_{i} \mathbf{H}_{0} \cdot \mathbf{n}_{i} \mathrm{~d} \Gamma .
$$

It must be pointed out that this formulation looks like a hybridization between finite-element method and an integral one. In (12), the first term leads to a sparse matrix $\mathbf{A}$ (similar to the one given by a FEM standard integration process) but the second term $\mathbf{B}_{s}$ is associated to an integral method. The obtained matrix system is so fully populated and compression algorithms must be used if problems with large numbers of elements are addressed. For instance, the use of the well-known fast multipole method (FMM) $[10,11]$ to compress Biot and Savart law is very efficient in such configuration [12].

Moreover, it must be pointed out that the integral (9) is computed only thanks to a surface integration of the averaged current distribution. This is an important difference with [9] where an accurate volume integration across the thickness is provided. This assumption can seem quite inaccurate especially for the computation of the element on itself when integration points and points where the field is expressed are closed. However, we will see with results obtained in the next section that the approach is not so inaccurate. On the other hand, we cannot compute the eddy-current losses. Indeed, this new method indicates only the field discontinuity through the shell, whereas the field on both sides is required for such a calculation.

\section{Numerical example}

Our new formulation has been implemented for 3D geometries and has been validated with two different academic examples. These examples are modeled by three different methods. The first one is the axisymmetric FEM using FLUX software [13]. The second one is the volume integral formulation proposed in [9]. The last one is the considered surface integral method.

\subsection{Hollow sphere}

In the first example, a conductive hollow sphere $(R=$ $0.1 \mathrm{~m}, e=2 \mathrm{~mm}, \sigma=6 \mathrm{E} 7 \mathrm{~S} / \mathrm{m})$ placed in a uniform axial magnetic field $\mathbf{H}_{0}=\left[\begin{array}{lll}0 & 0 & 1\end{array}\right](\mathrm{A} / \mathrm{m})$ (Fig. 2) is considered. We focus on the computed magnetic field in the air region close to the device (calculated along the path $\mathrm{AB}$ in Fig. 2) with both methods for both cases:

- Case 1: $f=50 \mathrm{~Hz}$, skin depth $\delta$ greater than the thickness $e(\delta=9.2 \mathrm{~mm}, \delta>e)$.

- Case 2: $f=4000 \mathrm{~Hz}$, skin depth $\delta$ smaller than the thickness $e(\delta=1 \mathrm{~mm}, \delta<e)$.

Results of the surface integral method are similar to those obtained with both axisymmetric FEM and integral methods with volume integration (Fig. 3). Because the external source field is the same on both sides $\left(\Gamma_{1}\right.$ and $\Gamma_{2}$ ) and on the average surface $\Gamma$, both integral methods give almost the same results. We can see small differences between computed values with our formulation and the reference (axisymmetric FEM) (Fig. 4). These differences can be explained by the infinite plane assumption and certainly by the use of a first-order triangular coarse mesh. As already mentioned, a disadvantage of this new integral formulation in comparison with existing ones is that it cannot be used to calculate eddy current loss.

\subsection{Thin conductive disk}

The second test case is a thin conductive disk $(R=1 \mathrm{~m}$, $e=20 \mathrm{~mm}, \sigma=6 \mathrm{E} 7 \mathrm{~S} / \mathrm{m}$ ) placed in a magnetic field $\mathbf{H}_{0}$ created by a current loop $(I=1 \mathrm{~A}, h=R / 4)$ (Fig. 5$)$. We have computed the magnetic field in the air region close to the device (calculated along the path CD in Fig. 5) with both methods for two cases:

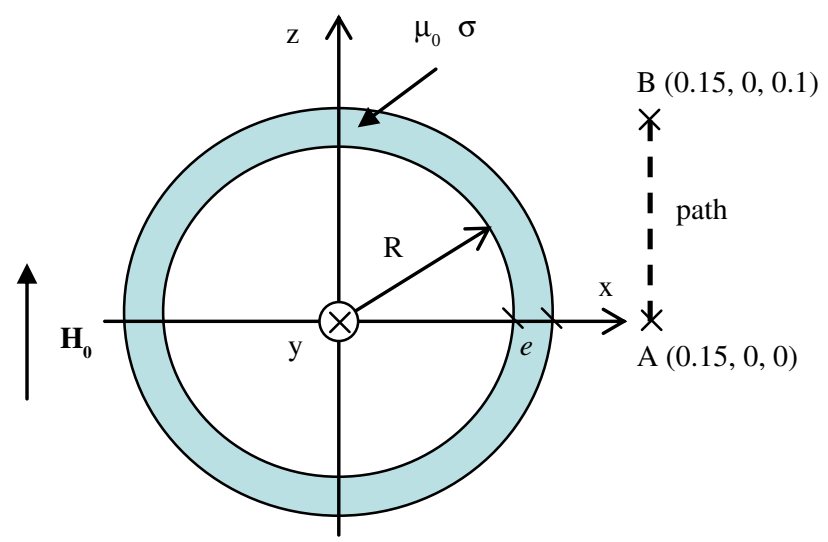

Fig. 2. Hollow sphere, notations and path where the external magnetic field is computed. 


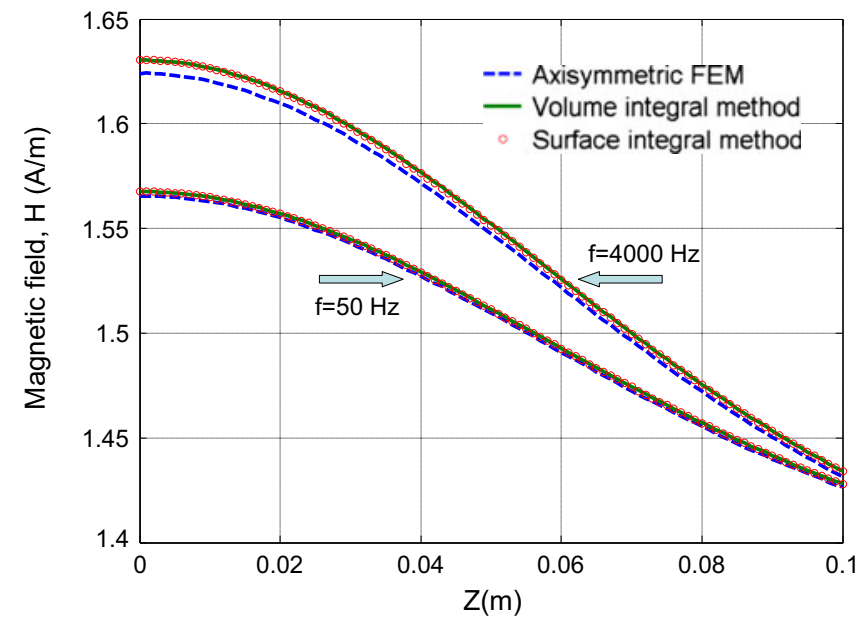

Fig. 3. Magnetic field along the path $\mathrm{AB}$ calculated by three methods in the fisrt example.

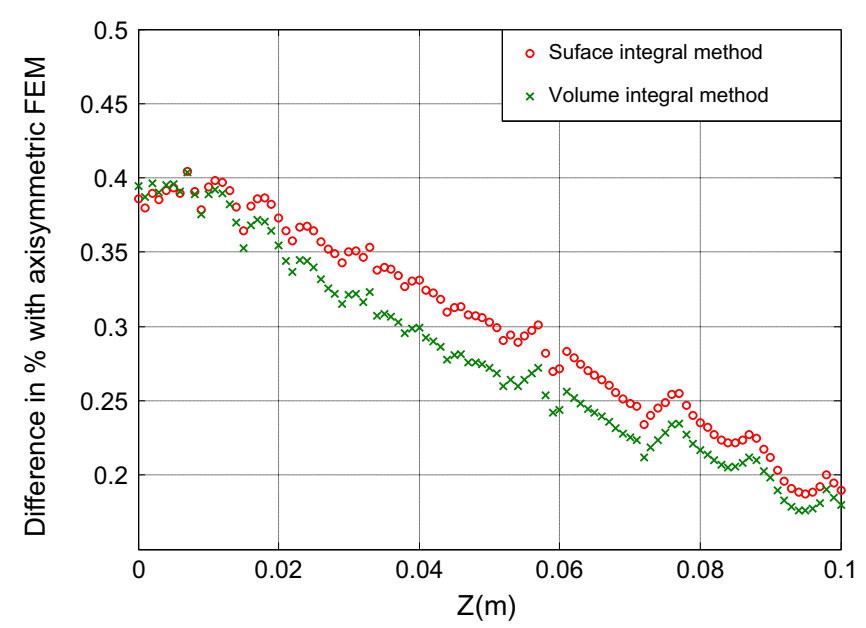

Fig. 4. Difference in $\%$ with the axisymmetric FEM solution for the magnetic field computation along line $\mathrm{AB}$ $(f=4000 \mathrm{~Hz})$.

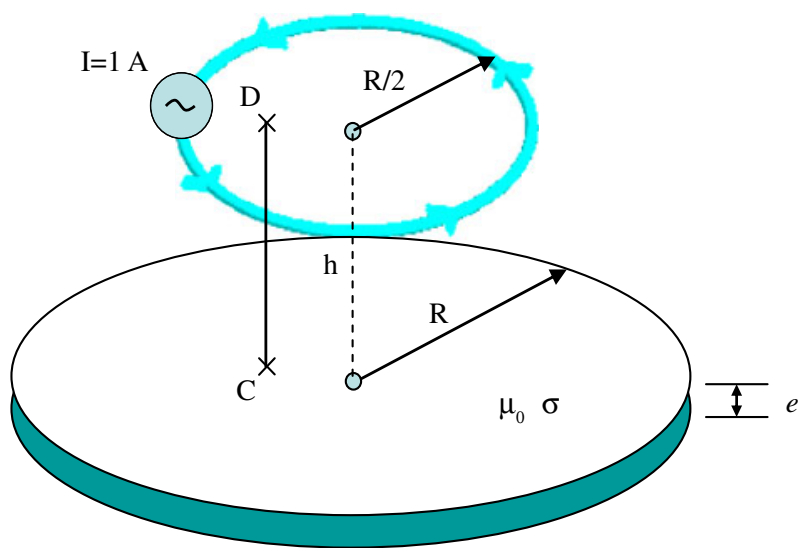

Fig. 5. Thin conductive disk, exciting coil and notations (C $(0.25,0,0.1), \mathrm{D}(0.25,0,0.25))$.

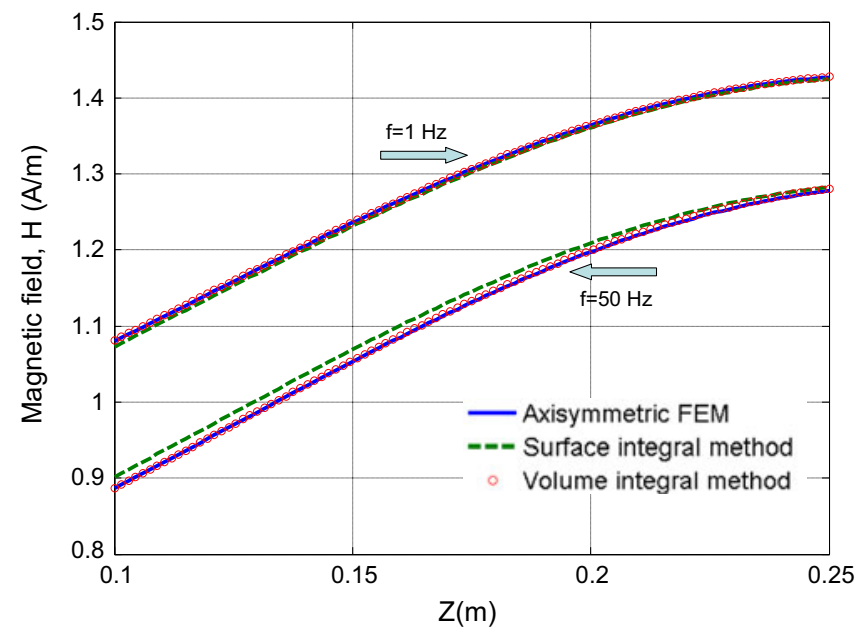

Fig. 6. Magnetic field along the path CD calculated by 3 methods in the second example.

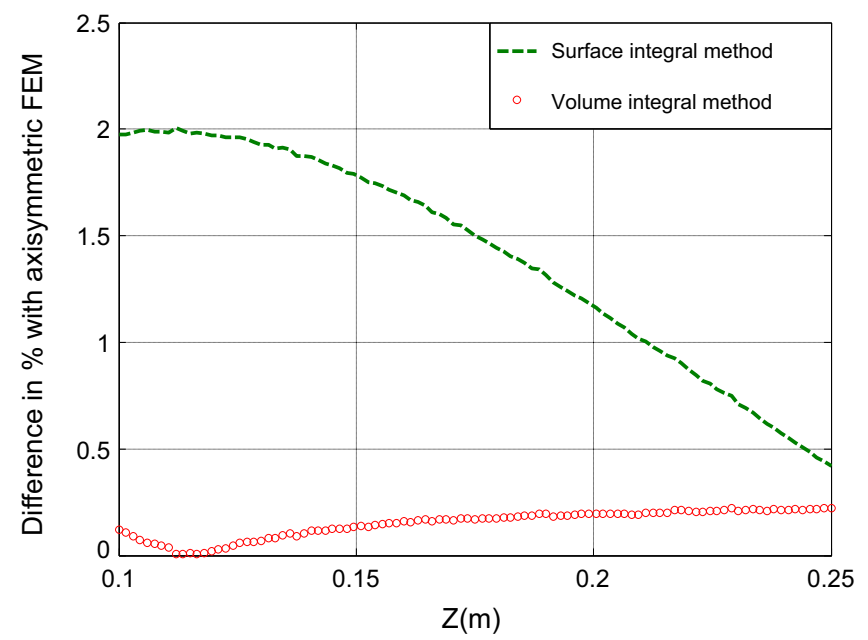

Fig. 7. Difference in \% with the axisymmetric FEM solution for the magnetic field computation along line CD $(f=50 \mathrm{~Hz})$.

- Case 1: $f=1 \mathrm{~Hz}$, skin depth $\delta$ greater than the thickness $e(\delta=65 \mathrm{~mm}, \delta>e)$ (Fig. 6).

- Case 2: $f=50 \mathrm{~Hz}$, skin depth $\delta$ smaller than the thickness $e(\delta=9.2 \mathrm{~mm}, \delta<e)$ (Fig. 6).

If we consider the axisymmetric FEM method as our reference, the integral method proposed in [9] leads to a maximal error of $0.1 \%$ at $f=1 \mathrm{~Hz}$ and $0.2 \%$ at $f=50 \mathrm{~Hz}$ (Fig. 7). The new integral method proposed in this paper presents a quite similar accuracy. In comparison with the finite-element method, the maximum differences are $0.6 \%$ at $f=1 \mathrm{~Hz}$ and $2 \%$ at $f=50 \mathrm{~Hz}$ (Fig. 7). In this case, let us notice that the formulation leads to less accurate results than in [9]. Moreover, Figure 7 shows that closer to the shell the post-processing point is, less the accuracy of the surface formulation is. These results can be explained by the integration of Biot and Savart law in equation (9) which is certainly less accurate while [9] takes properly into account the current density exponential decreasing 
Table 1. Memory space and CPU time (computer Intel CPU $5160 @ 3$ GHz; 16 GB RAM).

\begin{tabular}{ccc}
\hline Method & $\begin{array}{c}\text { CPU time } \\
(\mathrm{s})\end{array}$ & $\begin{array}{c}\text { Memory space } \\
(\mathrm{MB})\end{array}$ \\
\hline $\begin{array}{c}\text { Surface integral method } \\
\text { (3000 surface elements) }\end{array}$ & 170 & 144 \\
$\begin{array}{c}\text { Volume integral method }[9] \\
(3000 \text { surface elements })\end{array}$ & 1900 & 288 \\
\hline
\end{tabular}

law across the thickness. Moreover, the exact computation of the external source field on each side of the plate as in [9] can lead to more accurate result, in particular when source coils are close of the thin region.

Let us notice that the CPU time of surface integral method is reduced up to a factor of 10 in comparison with [9] (see Tab. 1) and the memory space is also reduced. This is due to a less important number of degrees of freedom and a simpler integration process. These last results demonstrate the advantage of the method.

\section{Conclusion}

In this paper, we have presented a new integral formulation using shell elements in order to model thin conductive non-magnetic regions. The formulation is general and various skin effects across thickness $(\delta>e$ or $\delta \approx e$ or $\delta<e)$ are taken into account. Moreover, it is very simple to implement in comparison with [9]. Even, if obtained results are a little bit less accurate close to the shell and losses cannot be computed, the formulation remains quite efficient if problems where only magnetic field in the air region is needed. The coupling of this formulation with compression algorithm seems to be promising in order to model real shielding applications. In further work, similar formulations enabling the modeling of thin conductive and magnetic shells will be developed.

\section{References}

1. L. Krähenbühl, D. Muller, IEEE Trans. Magn. 29, 1450 (1993)

2. A. Ospina, L. Santandrea, Y. Le Bihan, C. Marchand, J. Appl. Phys. 52, 23303 (2010)

3. C. Guérin, G. Tanneau, G. Meunier, P. Labie, T. Ngnegueu, M. Sacotte, IEEE Trans. Magn. 31, 1360 (1995)

4. L. Krähenbühl, P. Dular, T. Zeidan, F. Buretand, IEEE Trans. Magn. 40, 912 (2004)

5. O. Bíró, A. Bárdi, K. Preis, W. Renhart, K-R. Richter, IEEE Trans. Magn. 33, 1173 (1997)

6. C. Guérin, G. Meunier, IEEE Trans. Magn. 48, 323 (2012)

7. P. Thomas, Y. Le Menach, IEEE Trans. Magn. 48, 823 (2012)

8. V.Q. Dang, P. Dular, R.V. Sabariego, L. Krähenbühl, C. Geuzaine, IEEE Trans. Magn. 48, 407 (2012)

9. T. Le-Duc, G. Meunier, O. Chadebec, J-M. Guichon, IEEE Trans. Magn. 48, 427 (2012)

10. A. Buchau, S. Tsafak, W. Hafla, W. Rucker, IEEE Trans. Magn. 44, 1338 (2008)

11. R. Banucu, J. Albert, C. Scheiblich, V. Reinauer, W. Rucker, A. Hafla, A. Huf, IEEE Trans. Magn. 47, 1050 (2011)

12. T-T. Nguyen, T. Le-Duc, O. Chadebec, G. Meunier, J-M. Guichon, "Compression matricielle d'une formulation intégrale par la méthode multipôlaire rapide pour le calcul de courants de Foucault dans une région mince conductrice," in NUMELEC 2012, Marseille, France

13. Flux, Cedrat. Meylan, France, www.cedrat.com 